\title{
STEREO Superior Solar Conjunction Mission Phase
}

\author{
Daniel A. Ossing, Daniel Wilson, Kevin \\ Balon, Jack Hunt, Owen Dudley, \\ George Chiu, Timothy Coulter, Angel \\ Reese, Matthew Cox, Dipak Srinivasan, \\ Ronald Denissen \\ The Johns Hopkins University \\ Applied Physics Laboratory \\ 11100 Johns Hopkins Road \\ Laurel, MD 20723 \\ 240-228-8319 \\ Dan.Ossing@jhuapl.edu
}

\author{
David A. Quinn \\ NASA GSFC Code 444/584 \\ 8800 Greenbelt Rd \\ Greenbelt, MD 20771 \\ 301- 286-6681 \\ David.A.Quinn@nasa.gov
}

\begin{abstract}
With its long duration and high gain antenna (HGA) feed thermal constraint; the NASA Solar-TErestrial RElations Observatory (STEREO) solar conjunction mission phase is quite unique to deep space operations. Originally designed for a two year heliocentric orbit mission to primarily study coronal mass ejection propagation, after 8 years of continuous science data collection, the twin STEREO observatories entered the solar conjunction mission phase, for which they were not designed. Nine months before entering conjunction, an unforeseen thermal constraint threatened to stop daily communications and science data collection for 15 months. With a 3.5 month long communication blackout from the superior solar conjunction, without ground commands, each observatory will reset every 3 days, resulting in 35 system resets at an Earth range of $2 \mathrm{AU}$. As the observatories will be conjoined for the first time in 8 years, a unique opportunity for calibrating the same instruments on identical spacecraft will occur. As each observatory has lost redundancy, and with only a limited fidelity hardware simulator, how can the new observatory configuration be adequately and safely tested on each spacecraft? Without ground commands, how would a 3axis stabilized spacecraft safely manage the ever accumulating system momentum without using propellant for thrusters? Could science data still be collected for the duration of the solar conjunction mission phase? Would the observatories survive? In its second extended mission, operational resources were limited at best. This paper discusses the solutions to the STEREO superior solar conjunction operational challenges, science data impact, testing, mission operations, results, and lessons learned while implementing.
\end{abstract}

\section{TABLE OF ConTENTS}

1. INTRODUCTION 1

2. STEREO MisSiON AND THE CHALLENGE OF Solar Conjunction Mission Phase.

\section{Solar Conjunction Plan AND}

IMPLEMENTATION.

4. SCIENCE DATA RETURN .......................................... 8

5. OBSERVATORY CONFIGURATION .......................... 9

6. FAUlt PRotection MOdifications ................ 12
7. GUIDANCE AND CONTROL SUBSYSTEM

MODIFICATIONS ..............................................13

8. GROUND SYSTEM CONFIGURATION....................13

9. GROUND SYSTEM TESTING................................ 14

10. ON ORBIT OBSERVATORY TESTING AND LOSS OF COMMUNICATIONS WITH STEREO BEHIND..........15

11.POST CONJUNCTION ASSESSMENT.....................17

12.POST CONJUNCTION RESULTS ........................... 17

13. CONCLUSION...................................................19

ACKNOWLEDGEMENTS .........................................20

REFERENCES .........................................................20

BIOGRAPHY ........................................................... 20

\section{INTRODUCTION}

The STEREO mission and the challenges of the solar conjunction mission phase are described along with the adjustments required to continue science data return, necessary observatory and ground system changes, on-orbit observatory testing, and post conjunction results. As those that do not learn from history are doomed to repeat the mistakes, lessons learned are presented.

\section{STEREO Mission AND THE Challenge OF Solar Conjunction Mission Phase}

STEREO (Solar-TErestrial RElations Observatory) is the third mission in the Solar Terrestrial Probes program of the National Aeronautics and Space Administration (NASA) Science Mission Directorate Sun-Earth Connection theme. The mission consists of two nearly identical spacecraft in heliocentric orbits designed to investigate the threedimensional structure of the Sun's corona, the origin of coronal mass ejections (CME's), and the dynamic coupling between CME's and the Earth's environment. This Heliophysics mission is managed by NASA Goddard Space Flight Center (GSFC). 
The two spacecraft were designed, integrated, and operated by Johns Hopkins University/Applied Physics Laboratory (JHU/APL) with instrument suites provided by the Naval Research Laboratory, University of California Berkeley, University of New Hampshire, and the Observatoire de Paris. Each spacecraft is equipped with four instrument suites, as follows. Note that the IMPACT, PLASTIC, and SWAVES instruments measure data from the solar wind that passes by the spacecraft; they are referred to as the insitu instruments.

- Sun-Earth Connection Coronal and Heliospheric Investigation (SECCHI) - imaging

- In situ Measurements of Particles and CME Transients (IMPACT) - particle detectors

- Plasma and Suprathermal Ion Composition (PLASTIC) - particle detectors

- $\quad$ STEREO WAVES (SWAVES) - electric fields

\section{Orbits}

The two STEREO spacecraft were launched October 26, 2006 on a single launch vehicle into highly elliptical Earth orbits with apogee beyond lunar orbit. After several months, lunar flybys were employed to inject the spacecraft into heliocentric orbits, with STEREO Ahead's orbit slightly inside Earth's (and therefore slightly faster than Earth), and with STEREO Behind's orbit slightly outside Earth's (and therefore slightly slower than Earth). The resulting orbits cause the two satellites to move away from the Earth at a rate of $22^{\circ}$ per year. See Figure 1 below. Noting the direction of the Earth's revolution about the Sun, the positions of STEREO Ahead and STEREO Behind relative to the Earth gives rise to the nicknames "Ahead" and "Behind," respectively, for the two spacecraft.
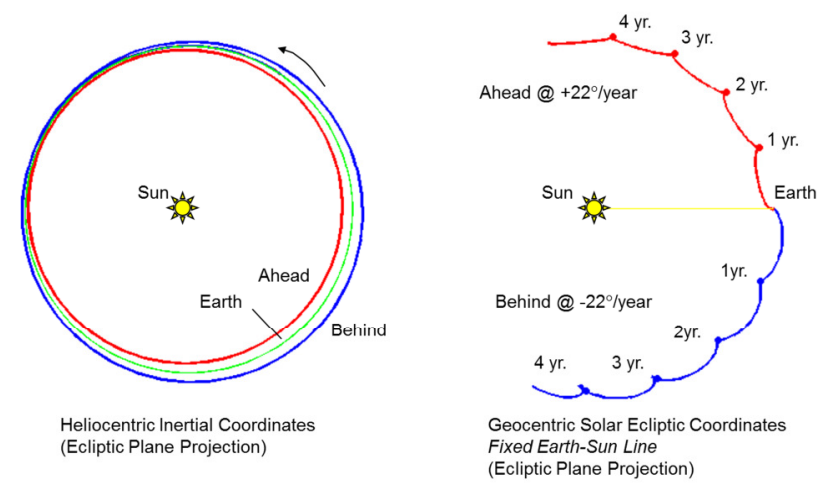

Figure 1. STEREO Orbits

As suggested by Figure 1, about eight years after heliocentric orbit insertion, the two spacecraft pass behind the Sun (superior solar conjunction). Although the angular radius of the Sun when seen from the STEREO orbits is only about $0.25^{\circ}, \mathrm{X}$-band communications are significantly degraded when the Sun-Probe-Earth (SPE) angle approaches $3^{\circ}$ due to solar scintillation effects. For planning purposes, the STEREO program picked the $2^{\circ}$ mark as the point to cease sending commands from the ground. It will take Ahead about 3.5 months to pass through this blackout region, and about 2 months for Behind.

\section{Command-Loss Timer}

STEREO is equipped with both a software command-loss timer (SCLT) and a hardware command-loss timer (HCLT). Both the timeout value and the corresponding action taken for the SCLT may be changed by mission operators. The SCLT may also be disabled. The HCLT is implemented in hardware/firmware. It cannot be disabled, and neither the timeout value nor the action taken can be changed after launch.

The STEREO HCLT expires if no command is received from the ground within three days (72.332 hours, to be precise). This value was chosen based on the requirements for the immediate post-launch mission phase, where orbital maneuvers might be required every few days in order to avoid Earth impact and to achieve eventual heliocentric orbit. The value is also consistent with the STEREO mission concept of operations, which calls for daily station contacts.

Among the many actions taken in response to an HCLT timeout, are:

- Shutdown of all four scientific instrument suites

- Avionics reset, including rebooting of the flight processors

- Reboot the transponder in the communications subsystem

- $\quad$ Power off the Star Tracker (ST)

- Adjust heaters for low-power consumption

As can be seen, collectively, these are drastic actions to take, and not without risk to spacecraft health. Therefore, an HCLT timeout is something to be avoided. In fact, in the almost 8 years of operation prior to initiation of solar conjunction planning, no HCLT resets occurred on either spacecraft. (We did, however, conduct several controlled commanded system resets - first to test the spacecraft safe mode, and later to activate a revised software application program. Commanded system resets are just as drastic.)

The STEREO HCLT design, while necessary and appropriate for early operations, presents a serious problem for surviving the solar conjunction. The Ahead spacecraft, for example, experiences 35 HCLT timeouts during its long 3.5 month communications blackout. 
Turning off the ST is particularly problematic. This action was included in the HCLT reset sequence because the spacecraft enters its safe mode (called Earth Acquisition, or EA mode) following a system reset, and safe mode does not require the ST. Instead, it relies on coarse Sun sensors and gyroscopes in the Inertial Measurement Unit (IMU) to maintain a power-positive sun-pointing attitude. Because of the aging of the STEREO gyros (see below), relying on gyros at this point in the mission is very risky. It is preferred to keep the ST powered on and locked onto stars through the system reset, if possible. But, by its very nature, the HCLT reset sequence cannot be changed. Hence, the solar conjunction plan must address this situation.

\section{Antenna Thermal Issue}

STEREO uses a $1.2 \mathrm{~m}$ parabolic dish as its High Gain Antenna (HGA). This is the spacecraft's primary means of communication. The HGA is gimbaled about the spacecraft Y-axis. The nominal attitude maintains the spacecraft $\mathrm{X}$ axis pointing at the Sun, and keeps the Earth in the spacecraft $X-Z$ plane, on the $-Z$ side. Thus, the HGA rotation axis is perpendicular to the Sun-Earth plane. By rotating the HGA gimbal, the flight software maintains the HGA boresight on the Earth as the spacecraft proceeds on its path around the Sun.

As the spacecraft approaches superior solar conjunction, sunlight falls more directly into the parabolic dish and gets concentrated at the focus. Even though the dish is flat black, this effect drives the antenna feed temperature to the point where damage is possible. See Figure 2. Specifically, after analyzing the materials on the antenna feed, it was concluded that degradation would start around $150^{\circ} \mathrm{C}$, and actual damage would occur around $170^{\circ} \mathrm{C}$. Projections indicate that if the HGA is allowed to point at the Earth through the solar conjunction, the temperature would greatly exceed the damage point.

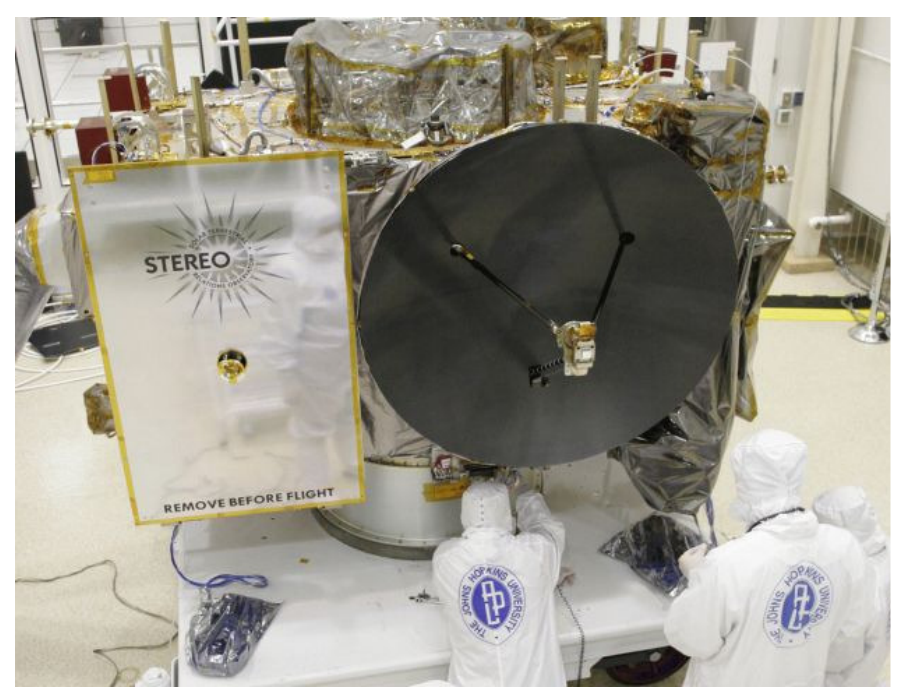

Figure 2. STEREO High Gain Antenna 1.2m parabolic dish
Moving the HGA boresight away from the Sun by just a few degrees has a dramatic beneficial effect on the feed temperature, but interrupts communications with Earth.

This thermal issue was not analyzed during the design phase because the goal for mission life was set at five years, and the issue did not arise until mission year eight. While it may seem short-sighted to stop the analysis at five years (and it is), this is typical of the type of management decisions that must be made in order to contain development costs.

The antenna thermal issue presents one of the biggest challenges for operating STEREO through the solar conjunction period. In fact, the issue presents a problem for many months before the actual conjunction and continues for many months after the conjunction. Altogether, the "hot zone" for HGA operations spans up to 15 months (447 days) on Ahead, and 13 months (397 days) on Behind.

\section{HGA Thermal Model}

In early April, 2014, monitoring of the HGA feed temperatures as a function of time showed temperatures increasing at an alarming rate in Figure 3:

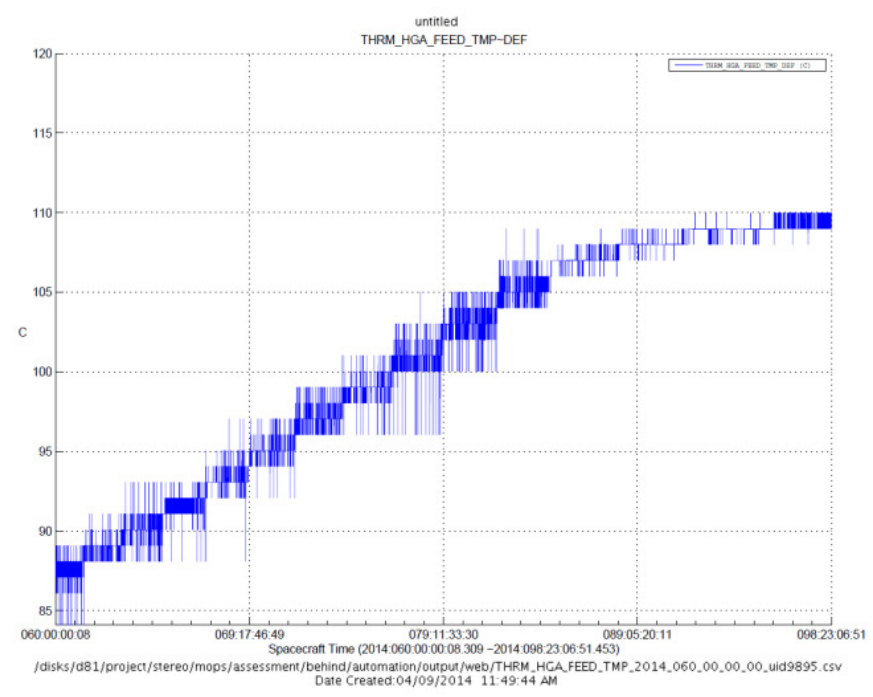

Figure 3. STEREO-B HGA feed temperature days 2014-000 through 096

Combining temperature telemetry with spacecraft ephemerides enabled examination of the direct relationship between HGA feed temperature and SPE angle. On looking at the data in this way, it was observed that as time moved forward, the SPE angle for both STEREO-A (red) and STEREO-B (blue) reduces, and temperature increases rapidly (towards the left on this chart). At this point, it became clear that temperatures would very quickly increase above the $136^{\circ} \mathrm{C}$ saturation limit of the temperature sensor, and perhaps beyond the $170^{\circ} \mathrm{C}$ limit at which damage to the hardware would become an issue. For this reason, it was 
agreed not to allow the HGA to get to temperatures above $125^{\circ} \mathrm{C}$. This would keep the antenna below the saturation limit, yet allow time to react should events warrant.

To better understand and predict this behavior, a thermal model of the HGA would be needed. The first step in creating a model was to take advantage of similar data from two spacecraft by normalizing both curves to a uniform distance of 1 AU (STEREO-A is in reality a bit closer to the Sun than 1 AU and STEREO-B is a bit further). Plotting those results revealed a virtual single curve dependent only on the HGA/SPE geometry:

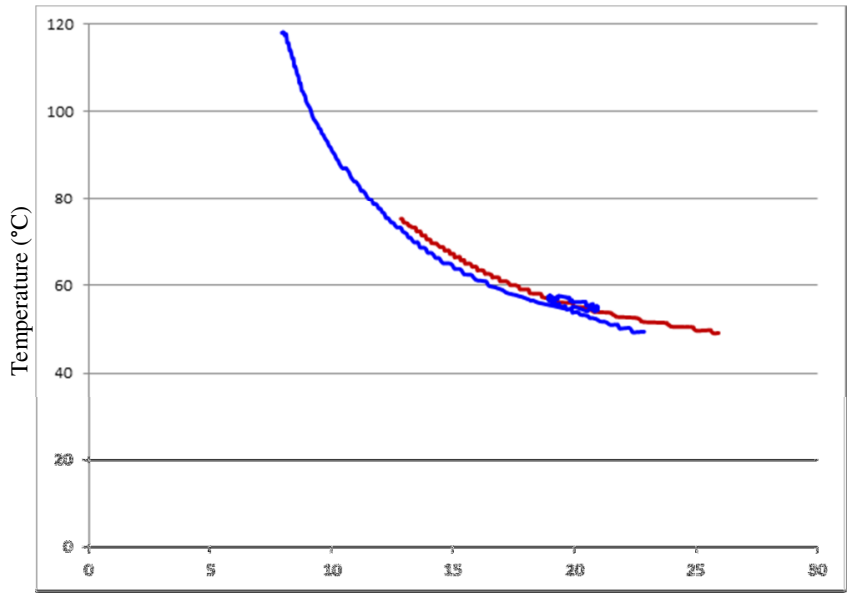

Sun-Probe-Earth Angle (degrees)

\section{Figure 3. HGA temperatures normalized to $1 \mathrm{AU}$ from Sun (STEREO-A (red) \& STEREO-B (blue))}

There was finite time for planning, since changing the orbit of either spacecraft was not an option. Clearly the HGA would have to be carefully managed, but as the primary means of communications with Earth, managing meant a reduction in data rates, if not a total loss of data for some period of time. The difficulty here would be to manage the HGA in such a way so as to minimize the effects of this solar focusing while maximizing the ability of both spacecraft to maintain contact with Earth - at as high a data rate as possible.

The question now would be how high would the temperature ultimately go? With no basis in the physics of the problem, extrapolations from curve fits were fine for short term predictions. However, without physics in the mix they would prove unreliable for long term planning.

Given the diameter and focal length of the STEREO HGA, as well as the focal plane assembly diameter and spacing from the HGA itself it would be possible to construct a first order thermal model of the HGA. When complete, the model would accommodate not only the rough solar focusing discussed earlier, but heating of the HGA dish by the Sun and the subsequent heating of the focal plane by IR radiation from the hot dish. Once delivered, the curve resulting from the model was added to the plot of the existing normalized data to confirm agreement in Figure 4:

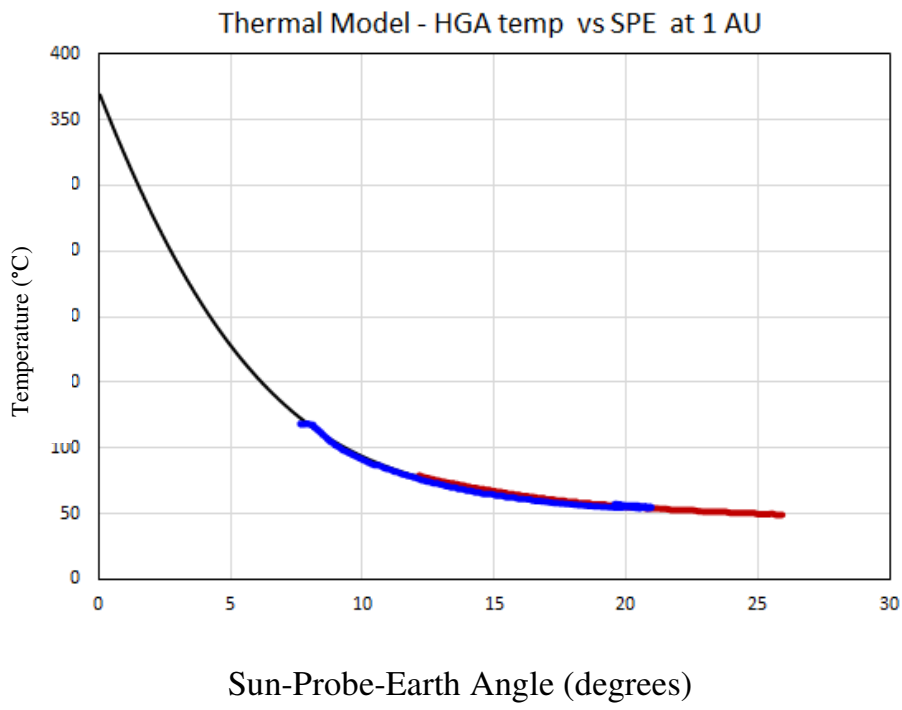

\section{Figure 4. Thermal model (black) with normalized STEREO-A (red) \& STEREO-B (blue) HGA temperatures}

With this, it would now be possible to adjust the curve for distance specific to each of the two spacecraft, which could be used in the time domain to allow future planning as conjunction activities moved forward. Once done, these relationships could be used to predict temperatures of the HGA for each spacecraft if the HGA were to keep pointing at Earth throughout the conjunction. In the case of STEREO-A, the HGA would experience temperatures above the predetermined $125^{\circ} \mathrm{C}$ comfort limit from August of 2014 through February of 2016, a period of well over a year. STEREO-B would violate the limit from November of 2104 through January of 2016, a few months less than STEREO-A, but a long time nonetheless. The next step would be to create a means of mitigating the problem.

\section{Side Lobe Operations and Radio Frequency Communications}

$(R F)$

After data analysis and testing on each spacecraft it was shown that moving the HGA boresight away from the Sun by just a few degrees had a dramatic beneficial effect on the feed temperature. By using the HGA side lobes, a level of RF communication can be maintained with the spacecraft and reduce thermal effects. Testing revealed that both the command and telemetry links can be maintained by switching from the main lobe of the HGA antenna pattern to either the first or second side lobes, located about $\sim 3^{\circ}$ and $\sim 6^{\circ}$, off the boresight. The big drawback to using the side lobes is the signal strength drops by 1 to 3 orders of magnitude. This forces the implementation of significantly reduced data rates and requires the use of NASA's Deep Space Network (DSN) 70 meter ground stations for daily contacts. 
The onboard software uses ephemerides for the spacecraft and Earth to compute the desired HGA gimbal angle. Because the signal strength on the side lobes is so low and because the widths of the lobes are thinner than that of the main lobe, it is important that the onboard software have reasonably accurate ephemerides. This requirement translates to the need to periodically update the onboard ephemerides during the many months of side lobe usage. This method for using the HGA side lobes was implemented and performed as predicted.

At low SPE angles, the Earth is near the edge of the low gain antenna's (LGA) hemi-spherical beam pattern. It was suggested that mission operations conduct a test to verify the LGAs signal would be receivable at low SPE angles. As the LGAs were not designed to operate at the 2 AU Earth range, their performance was tested on the Ahead observatory on June $7^{\text {th }} 2014$ at a SPE angle of $\sim 10.2^{\circ}$. The spacecraft was rotated twice around the spacecraft to Sun line. The first rotation characterized the signal for the $-Z$ LGA, and the second rotation characterized the signal for the $+Z$ LGA. The test results showed that both commanding and telemetry at very low data rates could be achieved during some portion of the rotation, see Figure 5.

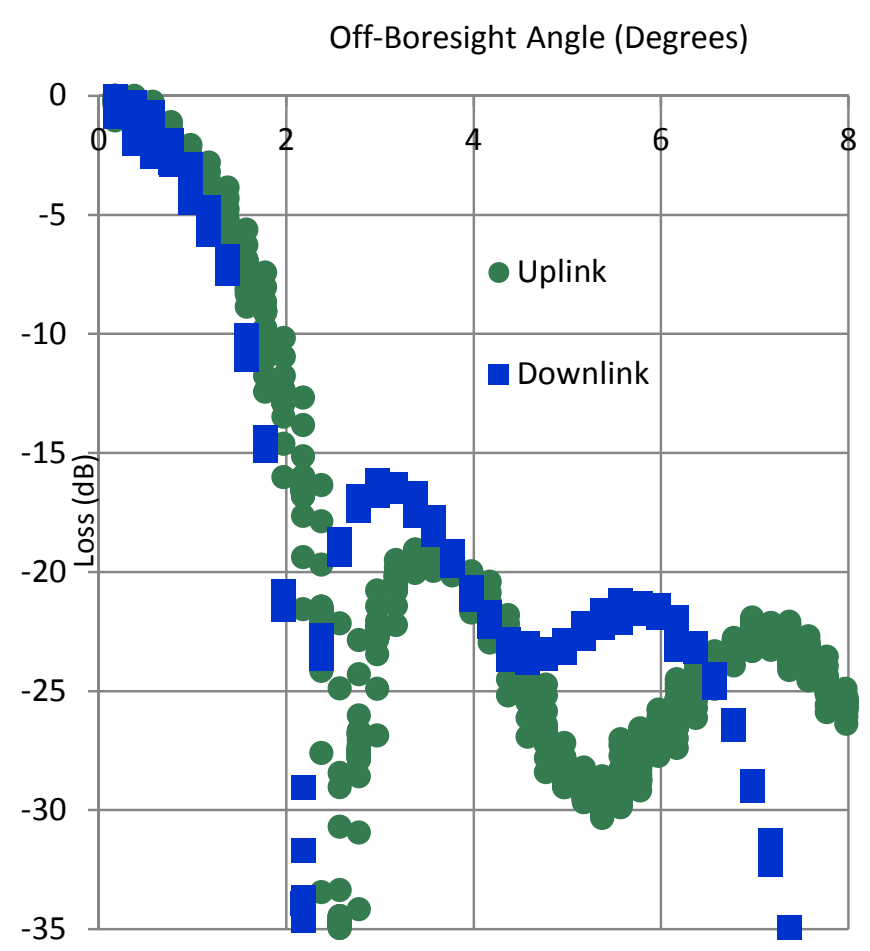

Figure 5. STEREO Ahead High Gain Antenna (HGA)
pattern, showing side lobes

As the SPE angle began to approach $0^{\circ}$ a steady increase in dropped frames and bit error rate was expected due to solar scintillation effects. Based on data from DSN, at the Xband frequencies, noticeable link degradation was expected as the SPE approached $3^{\circ}$. Around a $2^{\circ}$ SPE angle, the link degradation should be much more severe but the link could be closed depending on the degree of solar scintillation. Around a $1.5^{\circ} \mathrm{SPE}$ angle, the link was expected to be extremely hard to detect and maintain. The spacecraft was also going to be operating at lower data rates than initially thought due to the HGA feed temperature issue. Based on these assumptions, it was decided that the spacecraft would operate in solar conjunction mode while the SPE angle was less than $2^{\circ}$. In reality as the SPE decreased, the link performed both better and worse than originally estimated. It was observed that below $\sim 3.3^{\circ} \mathrm{SPE}$ angle the RF signal became very vulnerable to solar activity. When there was high solar activity within the line between the spacecraft and Earth, the signal degradation was much worse than expected. However when solar activity was fairly minimal, the signal degradation was much less than anticipated. As the SPE angle continued to decrease, solar activity became an increasing factor in the overall link degradation. At least some frames were able to be decoded on the HGA down to a $1.7^{\circ} \mathrm{SPE}$ angle while the spacecraft was rotating. Below this point, DSN was not tracking the spacecraft entering and exiting superior solar conjunction.

\section{Aging Spacecraft Components}

The longevity design requirement for STEREO was just two years, with a design goal of five years. Thus, both STEREO spacecraft have greatly exceeded expectations. Most hardware has shown no aging effects at all. However, the gyroscopes in the IMUs and the ST have some issues.

Each spacecraft is equipped with a pair of IMUs, with each IMU containing three orthogonal ring laser gyroscopes (RLGs) and three orthogonal accelerometers. The original operational concept was to fly with one IMU on at all times. The second IMU unit was to be kept powered off as a cold spare. In the event of an IMU fault, software onboard autonomously swaps to the backup IMU.

As gyros aged (and failed) the mission was forced to change to a new concept of operations called Reduced Gyro Operations (RGO). In RGO, the spacecraft "flies" on ST, sun sensors, and the Guide Telescope (essentially, a highprecision sun sensor), with both IMUs turned off. The (backup) IMU is turned on only for special events, such as comet observations or thruster-firing for momentum management. This changes the IMU duty cycle from $100 \%$ to less than $3 \%$, thus prolonging the remaining life of the IMUs.

The change required some adjustment of parameter values in the attitude determination and control software, as well as new onboard autonomy policies to maintain spacecraft health. For example, if the ST were to suddenly lose lock on stars, the system would need to autonomously power-up its only remaining fully-functional IMU.

STEREO Ahead switched to RGO in September 2013 and STEREO Behind in February of 2014. There was some observed degradation in pointing performance without gyro 
measurements, but the system was still able to meet or exceed its pointing requirements. From this point forward, both spacecraft were operating with one failed IMU, and one seriously aged IMU, using RGO to preserve the life of the remaining functional IMU.

Each spacecraft is equipped with a single ST. With the IMUs in a precarious state, the ST is relied upon much more than during the early years of the mission. By and large, the ST has performed extremely well. There have been a handful of cases, however, where the ST "dropped lock," i.e., failed to provide attitude solutions for extended periods of time (hours). In most cases, no root cause for the outages has been determined. In each case, eventually, the ST resumed normal operations. This phenomenon was dubbed the "lazy ST."

There is a known aging issue with this ST: its chargecoupled device (CCD) can accumulate radiation damage after years in space. The vendor had advised that lowering the CCD temperature can mitigate this problem. The ST is equipped with a Thermal Electric Cooler (TEC) to keep the ST at or below room temperature. The set point for the TEC can be changed by command. While there is insufficient data to make any statistically significant conclusion about the impact of possible radiation damage on the lazy ST behavior, lowering the TEC set point was an option if the lazy ST behavior became more than an occasional nuisance.

\section{Solar Conjunction Plan and IMPLEMENTATION}

The solar conjunction mission phase consists of three periods of operations; two side lobe operations periods, before and after solar conjunction, and the actual superior solar conjunction period. Side lobe operations are required for several months before and after solar conjunction to maintain the HGA feed assembly temperature to within measureable limits while maintaining some level of groundcommunications. The Table 1 lists the approximate duration of each period.

Table 1. Solar Conjunction Mission Phase Durations

\begin{tabular}{|c|c|c|c|}
\hline & $\begin{array}{c}\text { Pre-Solar } \\
\text { Conjunction } \\
\text { (days) }\end{array}$ & $\begin{array}{c}\text { Superior } \\
\text { Solar } \\
\text { Conjunction } \\
\left( \pm 2^{\circ}\right) \text { (days) }\end{array}$ & $\begin{array}{c}\text { Post-Solar } \\
\text { Conjunction } \\
\text { (days) }\end{array}$ \\
\hline $\begin{array}{c}\text { Ahead } \\
\text { Observatory }\end{array}$ & 216 & 105 & 126 \\
\hline $\begin{array}{c}\text { Behind } \\
\text { Observatory }\end{array}$ & 52 & 60 & 285 \\
\hline
\end{tabular}

As the STEREO mission was in its $8^{\text {th }}$ year of operations since launch, and NASA highly desired that the mission continue post solar conjunction, the following priorities, or "Laws of Solar Conjunction", were established by mission systems engineering to guide the schedule and use of the limited operational resources.

1. Take all reasonable precautions to keep the observatory safe.

2. Return to normal science operations as soon as reasonably possible. In cases where this law conflicts with the First Law, the First Law takes precedence.

3. Conduct science operations during conjunction, if reasonably feasible. In cases where this law conflicts with the first two, the first two laws take precedence.

Due to limited operational resources and the need to return to nominal science operations on the HGA main lobe as soon as possible, a goal for implementing this mission phase is to minimize operational changes to the system, i.e., procedures, ground software, memory objects, and daily cadence. This goal has the additional advantage of building upon well-tested and operationally-exercised systemconfigurations and procedures, while reducing some risk of introducing completely new system-configurations.

Differences between side lobe operations and nominal science operations primarily consist of the following:

- Lower data rates

- Daily 70 meter 3 hour supports to return limited science

- 34 meter supports, as available, to provide realtime space weather data

- Recording space weather for the in-situ instruments for the duration of the side lobe operations periods

- Re-partitioning the solid state recorder (SSR) for the duration of the solar conjunction mission phase

- $\quad$ Spacecraft telemetry playback only during each track, no science telemetry playback

- No instrument stored command buffer dumps during each track

- Load momentum dump commands the track before executing

In February of 2015, prior to the Ahead observatory entering solar conjunction, a project-level solar conjunction readiness review was held to evaluate the readiness of the project for conducting the STEREO Solar Conjunction mission phase. The review board consisted of NASA GSFC, science teams, critical STEREO spacecraft bus 
system leads, and mission operations managers. After action item adjudication, project approval to proceed was granted.

The entry and exit into the solar conjunction was at the $2^{\circ}$ SPE angle to ensure communication and verify the required observatory configuration. System momentum was set to 8 $\mathrm{Nms}$ to minimize the need for autonomous momentum dumps during the solar conjunction blackout period. Upon entry into the superior solar conjunction, each observatory was commanded, via an absolute timetag in the on board startup sequence, to rotate the spacecraft about the Sun-line at 5 degrees/minute to minimize system momentum buildup. As the 72.332 hour HCLT cannot be changed, each observatory undergoes a system reset every three days, thereby reinitializing the state of the observatory. As the SWAVES instrument uses minimal power and its default power-on configuration provides routine science, it remained on and was power-cycled with each three day HCLT system reset to continue to record science data at 1 packet per minute.

Upon exiting the solar conjunction blackout period at $2^{\circ}$ SPE angle, each observatory was commanded, via an absolute timetag in the on board startup sequence, to return to the nominal 3-axis stabilized attitude. The Ahead observatory was recovered from solar conjunction in early
July of 2015 on the $2^{\text {nd }}$ side lobe of the HGA after it reached the $2^{\circ}$ SPE angle. Daily DSN communications on the $2^{\text {nd }}$ side lobe were resumed using an eight hour daily track for six weeks to playback the spacecraft data for analysis. Instruments were powered on and reconfigured for continuing limited real-time science, and the IMPACT and PLASTIC in-situ instruments resumed recording space weather at the one packet per minute rate.

In November of 2015 the Ahead observatory returned back to the main lobe of the HGA, at a $1^{\circ}$ offset which it rode along to the center of the main lobe which was reached at the end of December. All of the data on the SSR was played back twice to ensure completeness, the SSR was repartitioned back to the nominal science configuration. Note that as Earth Acquisition mode has been discontinued, see section 4, there was an additional 108.7 Mbits in three Earth Acquisition mode SSR partitions which was re-allocated to the instrument partitions.

A timeline depicting the significant events for each observatory during the solar conjunction mission phase is shown in Figures $6 \& 7$. Note that for comparison, the timeline scale is the same for both observatories.

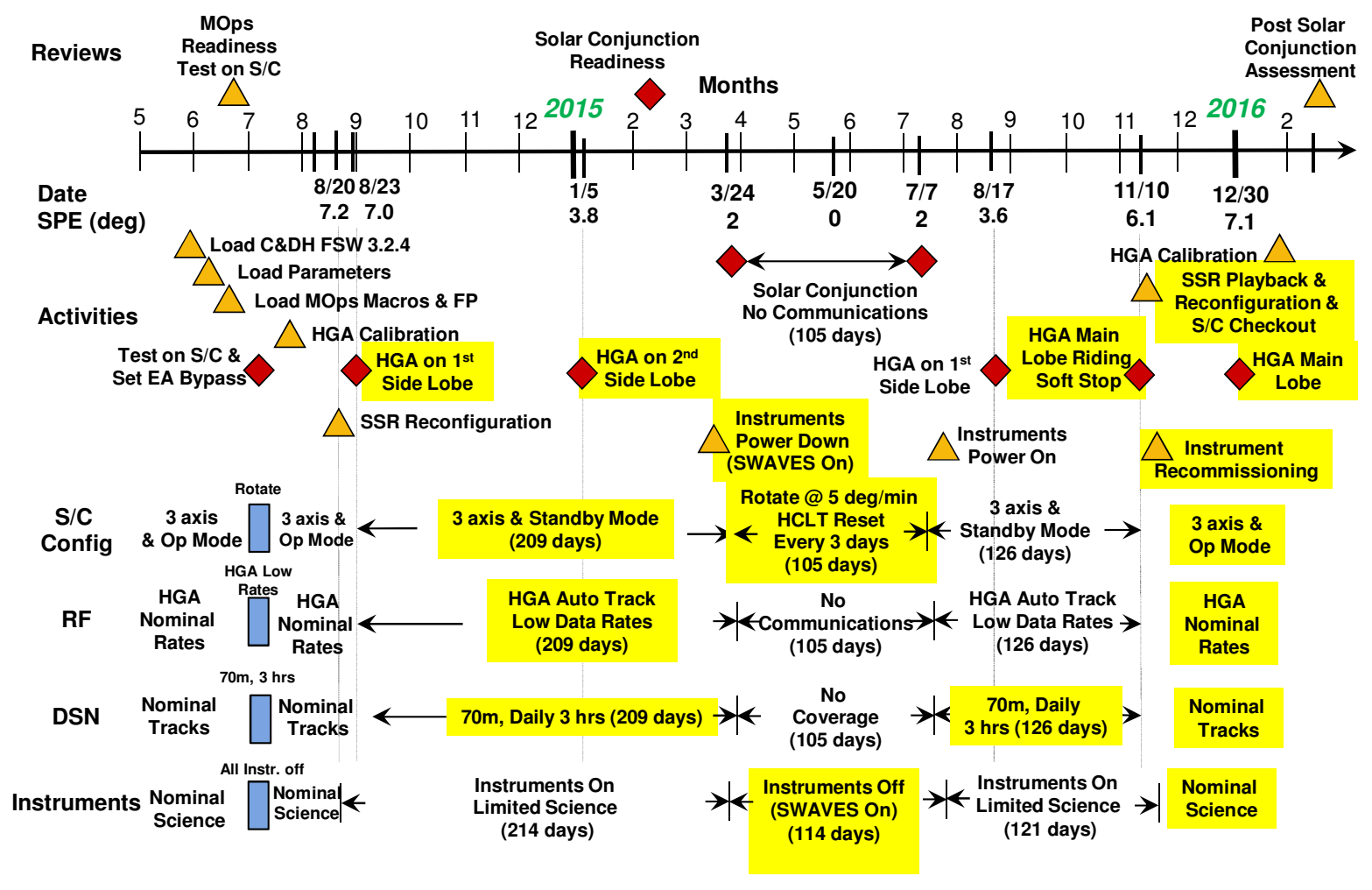

Figure 6. STEREO Ahead Timeline for Superior Solar Conjunction 


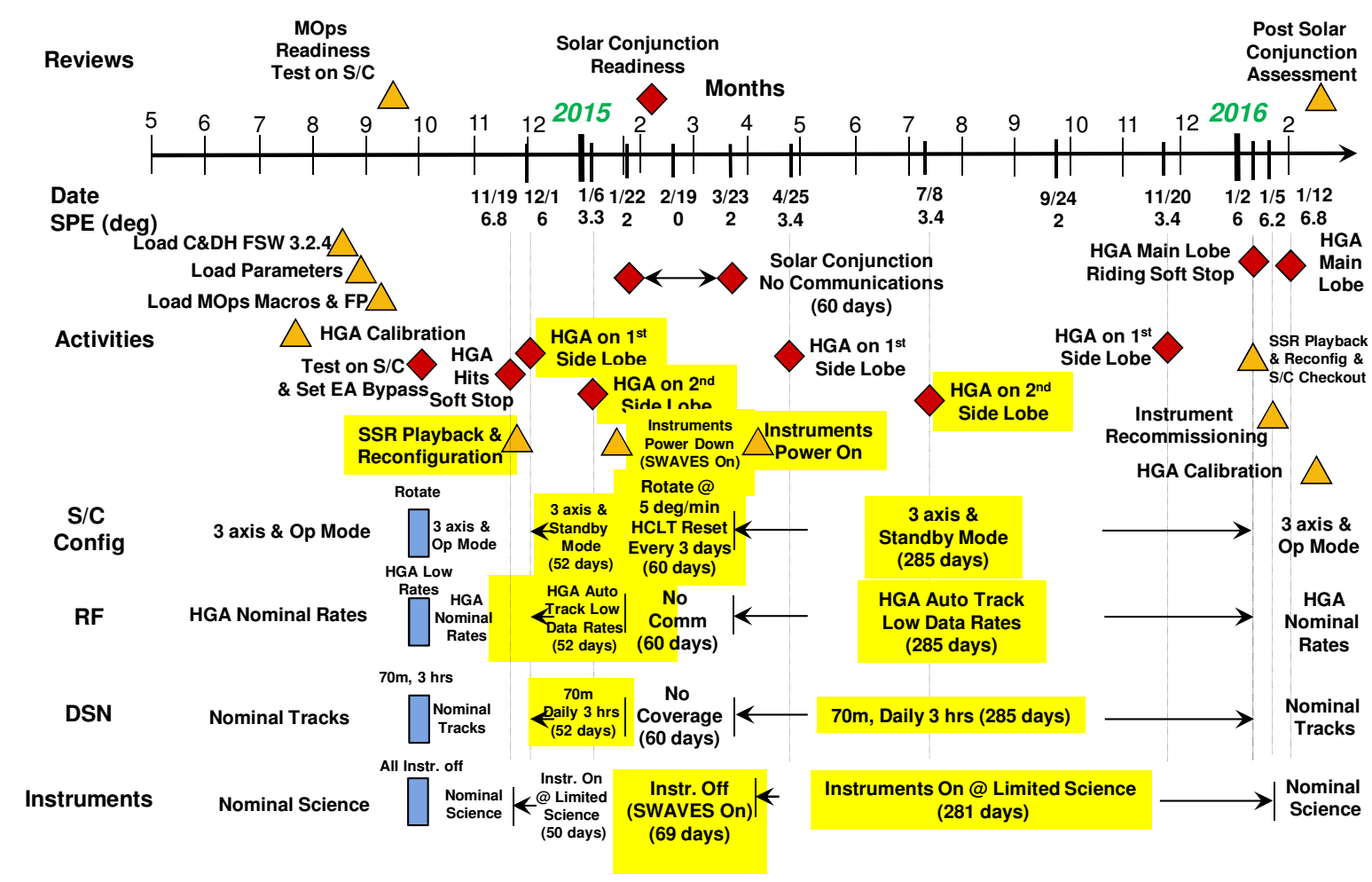

Figure 7. STEREO Behind Timeline for Superior Solar Conjunction

\section{SCIENCE DAta Return}

While off-pointing the HGA to protect the feed assembly from damage, there is a greater than $20 \mathrm{~dB}$ loss in RF signal levels during side lobe operations. This necessitates using lower data rates for daily communications. Using the three DSN 70 meter stations, through testing, the sweet spot for the optimal uplink and downlink signals were selected to provide a $6 \mathrm{~dB}$ uplink margin and $2 \mathrm{~dB}$ downlink margin as listed in Table 2. The downlink rates for the 70 meter stations still allow for limited real-time science data return. The DSN 34 meter subnet, including the three European Space Agency (ESA) deep space stations, can also be used for daily limited real-time science data return, spacecraft state of health checks, and anomaly recovery, at very low downlink rates. Note that the ranging performance is low on side lobe operations and intermittent loss of ranging lock did occur. Also, ranging was disabled during $34 \mathrm{~m}$ DSN supports to provide additional downlink margin.

Table 2. STEREO Side Lobe Data Rates

\begin{tabular}{|c|c|c|c|c|c|c|}
\hline & \multicolumn{2}{|c|}{70 meter } & \multicolumn{2}{|c|}{34 meter } & \multirow[b]{2}{*}{$\begin{array}{c}\text { HGA } \\
\text { Angle Bias } \\
\text { (deg) }\end{array}$} & \multirow[b]{2}{*}{$\begin{array}{l}\text { Ranging Mod } \\
\text { Index (rads) }\end{array}$} \\
\hline & $\begin{array}{c}\text { Downlink } \\
\text { Rate } \\
\text { (kbps) }\end{array}$ & $\begin{array}{c}\text { Uplink } \\
\text { Rate (bps) }\end{array}$ & $\begin{array}{c}\text { Downlink } \\
\text { Rate } \\
\text { (kbps) }\end{array}$ & $\begin{array}{c}\text { Uplink } \\
\text { Rate (bps) }\end{array}$ & & \\
\hline Ahead $1^{\text {st }}$ Side Lobe & 10 & 500 & 1 & 125 & 3.2 & 0.305 \\
\hline Ahead $2^{\text {nd }}$ Side Lobe & 3 & 125 & 0.633 & 125 & 6.1 & 0.61 \\
\hline Behind $1^{\text {st }}$ Side Lobe & 10 & 500 & 1 & 125 & 3.4 & 0.305 \\
\hline Behind $2^{\text {nd }}$ Side Lobe & 3 & 125 & 0.633 & 125 & 6.2 & 0.61 \\
\hline Nominal Rates (Main Lobe) & 720 & 2000 & 160 & 2000 & 0 & 0.305 \\
\hline
\end{tabular}

With the low downlink rates and nominal 3 hour 70 meter daily supports, spacecraft housekeeping generation rates needed to be reduced. As the data return concept records all spacecraft telemetry packets generated and it was highly desired to minimize changes to the spacecraft and ground configuration for solar conjunction operations, real-time spacecraft housekeeping telemetry rate was reduced by $95 \%$ to $0.14 \mathrm{kbps}$.

The SSR was repartitioned to allow for recording spacecraft housekeeping during side lobe and solar conjunction operations, for 335 days (maximum $2^{\text {nd }}$ side lobe duration). 
The SSR capacity was split with in-situ instruments receiving half for recording space weather data for the duration of side lobe operations at one packet per minute each and half for spacecraft housekeeping. Due to the large recording volume necessary for images, the SECCHI instrument did not record any data for the solar conjunction mission phase, only real-time science was provided. To minimize the impact of the changes on the operational system, the same number of partitions was maintained by zeroing out unused partitions.

To maintain the health and safety of the observatories, the daily spacecraft housekeeping volume is 11.9 Mbits which requires the following playback durations each day using the side lobe downlink rates:

- 34 minutes to playback @ $10 \mathrm{kbps}$

- 3 hours (or track duration) @ 3 kbps

As the recorded science volume cannot be supported with the side lobe downlink rates, daily SSR playbacks of science data are not possible until the return to the HGA main lobe. To allow the in-situ instrument teams to monitor the recording of the space weather data for the possible 16 month duration of the side lobe operations, the SSR Prediction data product was modified to display the current percent full of each partition.

Initially, the instruments were to be off during the side lobe operations as the spacecraft and Mission Operations Center (MOC) were not setup for collecting, returning, and processing science data at low data rates. Once Ahead solar conjunction spacecraft testing was completed in July 2014, science telemetry allocations were coordinated with the science teams. The instrument teams have the following real-time telemetry allocations during side lobe operations:

- $\quad 1^{\text {st }} \mathrm{HGA}$ side lobe $=7.4 \mathrm{kbps}$ of $10 \mathrm{kbps}$ data rate

- $\quad 2^{\text {nd }}$ HGA side lobe $=1.7 \mathrm{kbps}$ of $3 \mathrm{kbps}$ data rate

Note that the $1^{\text {st }}$ side lobe allocation includes an allocation for playing back 48 hours of spacecraft SSR volume.

In Table 3 is the real-time instrument telemetry rates used during side lobe operations. Note that for the first side lobe only, SWAVES and IMPACT alternated each week as to which receives the additional 385 bps. This was implemented in two separate downlink configuration macros ${ }^{1}$ and on board tables.

Due to the low real-time downlink rates, the IMPACT Solar Wind Electron Analyzer (SWEA) instrument remained off for the duration of the side lobe operations.

1 A macro is a sequence of commands to accomplish a higher level function, i.e., SSR playback, configure for downlinking at $720 \mathrm{kbps}$, or autonomy rule safing response.
After the Ahead observatory began side lobe operations, to increase science data return during side lobe operations, the first and second side lobe communications were tested with the DSN 34 meter subnet and ESA 35 meter stations (first side lobe only with ESA). It was determined that the 633 bps/125 bps downlink/uplink rates met the $6 \mathrm{~dB}$ uplink margin and $2 \mathrm{~dB}$ downlink margin requirement on the first side lobe, however, there is only $3 \mathrm{~dB}$ margin on the uplink on the $2^{\text {nd }}$ side lobe.

While the current space weather table on board for downlinking at 633 bps contains only instrument data and spacecraft data is required for monitoring bus state of health during each track, two new macros and a new table were developed. This provided the usual one packet per minute of space weather for each in-situ instrument, $346 \mathrm{bps}$ for SECCHI, and 140 bps for spacecraft housekeeping. This new table was also used in between DSN tracks during side lobe operations. Note that SSR playback did not occur during 34/35 meter supports as the downlink rate is too low for it to be useful.

Use of the DSN 34 meter subnet and ESA 35 meter stations results in use of 13 additional antennas to provide not only increased science data return along with the daily 70 meter supports, but also provided for uplink coverage when no 70 meter stations were available.

Before entering solar conjunction, the instruments were powered off, except for SWAVES, and the SSR pointers reset. The Data Summary Table, which records the maximum and minimum of all analog voltage, current, temperature, and pressure telemetry, was dumped and reset.

\section{ObServatory CONFiguration}

\section{HCLT Enforcement of Safe Mode during Solar Conjunction}

During extended periods of communications blackout, the HCLT times out repeatedly at intervals of 72.332 hours, with each timeout triggering a full system reset and transition to Safe Mode. Throughout the blackout period during the solar conjunction - altogether 105 days for STEREO Ahead, 60 days for STEREO Behind - the STEREO Ahead observatory undergoes 35 system resets and transitions to Safe Mode, and STEREO Behind undergoes 20 system resets and transitions to Safe Mode. Because of this frequent and periodic enforcement of Safe Mode configuration during solar conjunction, the only practical means of controlling the observatory configuration during solar conjunction is to reengineer the observatory's Safe Mode configuration itself.

\section{Replacing “Earth Acquisition” Safe Mode}

In the STEREO observatories' original fault protection design, the system reset function mainly consisted of a sequence of field-programmable gate array (FPGA) encoded 
Table 3. STEREO Instrument Real-time Telemetry Rates during Solar Conjunction Mission Phase

\begin{tabular}{|l|c|c|c|c|c|c|}
\hline Resource & IMPACT & PLASTIC & SECCHI & SWAVES & Total & Allocation \\
\hline $\begin{array}{l}\text { On during side-lobe } \\
\text { operations? }\end{array}$ & Yes & Yes & Yes & Yes & & \\
\hline $\begin{array}{l}\text { On during superior } \\
\text { conjunction? }\end{array}$ & No & No & No & Yes & & \\
\hline SSR usage (Gbits) & 1.253 & 1.253 & 0 & 1.645 & 4.151 & 4.294 \\
\hline Side lobe 1 & & & & & & \\
\hline $\begin{array}{l}\text { SWAVES Prime R/T } \\
\text { on side-lobe 1 (kbps) }\end{array}$ & 0.679 & 0.6673 & 5.41 & 0.460586 & 7.216886 & 7.22 \\
\hline $\begin{array}{l}\text { IMPACT Prime R/T } \\
\text { on side-lobe 1 (kbps) }\end{array}$ & 1.064 & 0.6673 & 5.41 & 0.072533 & 7.213833 & 7.22 \\
\hline Side lobe 2 & & & & & & \\
\hline $\begin{array}{l}\text { R/T on side-lobe 2 } \\
\text { (kbps) }\end{array}$ & 0.328 & 0.184 & 1.075 & 0.072533 & 1.659533 & 1.658 \\
\hline
\end{tabular}

power switching actions capable of reliably reconfiguring the spacecraft even if there is no functioning flight processor. Part of this hardware sequence triggers boardlevel resets of the two onboard processors - the Control and Data Handling (C\&DH) Processor, which controls the MILSTD-1553 data bus, and the Guidance and Control (G\&C) Processor. Both processors subsequently boot, load, and initiate their respective operational flight software programs that are stored in Electrically Erasable Programmable ReadOnly Memory (EEPROM).

Whereas the G\&C Processor is only capable of running the dedicated G\&C software application to support spacecraft operations, two independent software applications are available to run on the C\&DH Processor - the C\&DH software application and the EA software application either of which may be loaded and initiated on the C\&DH Processor. The C\&DH software application is the principal operating software for the C\&DH Processor, and is used to support all STEREO commissioning and science operations requirements. The EA software application is the fallback in case of a critical system fault or failure. From the beginning of the STEREO mission, the C\&DH Processor had been configured to load and initiate the EA software application in response to a system reset.

Within the context of the STEREO observatories' singlestring redundancy architecture, the EA software application allows the C\&DH Processor to perform all essential Safe Mode functions even if the G\&C Processor has failed. However, compared to the combined functionality offered by the $C \& D H$ and $G \& C$ Processors running $C \& D H$ and G\&C software applications, respectively, the EA software application provides limited functionality, having no support for HGA pointing and no support for routing of instrument commands and collection of instrument telemetry.

Because the EA application software requires the usage of IMU measurement data for control of spacecraft attitude, the new constraint against excessive IMU on-time dictates the abandonment of EA application software in advance of the solar conjunction, and for the remainder of the STEREO mission.

Consequently, the first step in reengineering STEREO's Safe Mode for solar conjunction is to reconfigure the C\&DH Processor to load and initiate the C\&DH software application instead of the EA software application in response to a system reset. This change in the STEREO boot loader software behavior is implemented by setting an avionics hardware logic discrete that the boot loader uses to determine which operational software to load into C\&DH processor random-access memory (RAM).

\section{New "Solar Conjunction" Safe Mode}

The remaining engineering problem to solve is to utilize the available C\&DH software functionality to establish the desired observatory configuration for solar conjunction operations. Following each system reset, the C\&DH software is capable of automatically running preprogrammed macro command sequences that have been stored in C\&DH processor EEPROM. Such macro command sequences may include unconditional command 
sequences executing upon software initiation, or conditional command sequences triggered by autonomy rules ${ }^{2}$ or timetag rules. These software-executed command sequences thus are the last component of an overall system reset sequence that includes the following (in order):

a. A non-modifiable FPGA-encoded sequence of power switching actions, originally designed to reconfigure the observatory for EA Mode operations.

b. Board-level resets of the $\mathrm{C} \& \mathrm{DH}$ and G\&C Processors, with the $\mathrm{C} \& \mathrm{DH}$ Processor reconfigured to load and initiate the C\&DH software application instead of the EA software application.

c. Stored programmable command sequences executed by the $C \& D H$ software application to further reconfigure the observatory from the EA Mode configuration to a new "Solar Conjunction" Safe Mode configuration supporting solar conjunction operations.

\section{Active $G \& C$ Actuators}

As in all observatory modes, attitude control is maintained using four reaction wheels, except when dumping momentum, when thrusters are used for up to several minutes. "Solar Conjunction" Safe Mode retains the ability to point the HGA at the Earth, which is necessary to resume communications post-solar conjunction as LGA communications are marginal due to solar scintillation interference effects and the 2 AU distance. As the FPGA hardware sequence powers off the HGA Rotary Actuator (HGARA) during each system reset, it was therefore necessary to power the HGARA back on using the autoexec macro command sequence.

\section{Spacecraft Attitude Control Mode}

To evenly distribute the solar pressure body torques and minimize the build-up of system momentum throughout solar conjunction, the G\&C software is commanded during solar conjunction to a "rotisserie" mode, rotating slowly at 5 degrees per minutes about the spacecraft-Sun line. This reduces the likelihood of unobserved autonomous thruster firings during the solar conjunction period.

$\mathrm{G} \& \mathrm{C}$ rotisserie mode is initiated and terminated via absolute timetag/macro command sequences so that rotisserie mode is only in effect during the predicted solar-conjunction communications blackout period. Following each HCLTinitiated system reset, $\mathrm{G} \& \mathrm{C}$ is commanded to rotisserie mode if the onboard coordinated universal time (UTC) clock has passed the solar conjunction entry time (for STEREO Ahead, this is set to 00:00:00z on March 24, 2015). HGA steering is disabled whenever the spacecraft is in rotisserie mode. $\mathrm{G} \& \mathrm{C}$ is similarly commanded back to

\footnotetext{
${ }^{2}$ Autonomy rules are uploadable data structures that work with an on board rule engine to implement "IF-THEN" logic. For example, IF an instrument temperature reading is above $50^{\circ}$ for 10 seconds, THEN turn it off.
}

nominal 3-axis inertial attitude control (with HGA autosteering enabled) if the onboard clock has passed the solar conjunction exit time (for STEREO Ahead, this is 00:00:00z on July 7, 2015).

Outside of the solar conjunction blackout period, the G\&C attitude control mode is determined based on the antenna in use. When the RF signal path is switched to the HGA or the $-\mathrm{Z}$ LGA (on the same side of the spacecraft as the HGA), G\&C is commanded to nominal 3-axis inertial attitude control with HGA auto-steering enabled. When the RF signal path is switched to the $+\mathrm{Z}$ LGA (on the opposite side of the spacecraft as the HGA), G\&C is commanded to the $5^{\circ}$ /minute rotisserie mode with HGA steering disabled.

\section{Antenna Selection}

The FPGA hardware reset sequence unhelpfully switches the RF signal path to the $+Z$ LGA following each System Reset. To establish the desired HGA configuration for the solar conjunction blackout period, commands switching the $\mathrm{RF}$ signal path from the $+\mathrm{Z}$ LGA to the HGA were added to the autoexec macro command sequence.

Supplemental to the initial HGA configuration enforced periodically at 72.332 hour intervals, an antenna roundrobin sequence was incorporated, such that at 24 hours after each system reset, the RF signal path is switched from the HGA to the $-Z$ LGA, and at 48 hours after each system reset, the path is switched from the $-Z$ LGA to the $+Z$ LGA. The 24-hour round-robin switchover to the $-Z$ LGA is implemented via relative timetag rule/macro command sequence, with the triggering timetag loaded as part of the autoexec macro command sequence, then executing after a "relative" delay of 24 hours. The 48-hour round-robin switchover to the $+\mathrm{Z}$ LGA is implemented via autonomy rule/macro command sequence, conditional on no uplink commands being received for 48 hours. This autonomy rule/macro sequence is part of the fault protection system, and was inherited unchanged from the long-term science operations mode.

\section{$X$-Band Transponder}

The FPGA hardware reset sequence performs a hardline reset of the Small Deep Space Transponder (SDST) during each system reset, after which stored macro command sequences are used to establish one of two configurations used for solar conjunction. The first configuration is the default emergency mode, with the uplink set for $7.8125 \mathrm{bps}$ non-return-to-zero level (NRZ-L) and the downlink set for 11.712 NRZ-L, Turbo rate $=1 / 6$ coding, and is used when the selected antenna is either the " $Z$ " or " $+Z$ " LGA. The second configuration consists of an uplink set for 125 bps NRZ-L and the downlink set for 633 bps B-Phase-L, Turbo rate $=1 / 6$ encoding, and is used when the selected antenna is the HGA. The SDST configuration changes are implemented alongside the antenna round robin transitions.

Traveling Wave Tube Amplifier (TWTA) 
With each system reset, the FPGA hardware sequence demotes the TWTA to a non-transmitting Standby Mode as part of its load shedding function. A conditional autonomy rule/macro command sequence is responsible for turning on TWTA transmissions if battery pressure is over a minimal threshold for 230 seconds. This autonomy rule/macro sequence is part of the fault protection system, and was inherited unchanged from both the legacy EA Mode and the long-term science operations mode.

Analysis of the loss-of-communications anomaly on STEREO Behind revealed there to be no onboard mechanism for automatically powering on the TWTA if some unanticipated failure were to leave it switched off. This situation would leave the spacecraft unable to broadcast a downlink signal without the intervention of ground-station uplinked commands. To fill this gap in functionality, commands powering on the TWTA box and the Electronic Power Conditioning (EPC) filament were added to the autoexec macro command sequence.

\section{Science Instruments}

As part of the load-shedding function of each system reset, the FPGA hardware reset sequence switches off the operational power of all science instruments, while switching on their associated survival heaters. For solar conjunction, the SWAVES instrument is powered back on (and the survival heater powered back off) to continue collecting science data at a reduced one-packet-per-minute cadence throughout the solar conjunction period.

As SWAVES science gathering was to be accommodated during solar conjunction but otherwise not supported for safe mode situations beyond solar conjunction, the SWAVES power-on was implemented via conditional command sequence rather than being incorporated directly into the autoexec macro command sequence. Specifically, this functionality was attached to the absolute timetag/macro command sequence used to initiate $\mathrm{G} \& \mathrm{C}$ rotisserie attitude control following each system reset if the onboard UTC clock has passed the solar conjunction entry time (for STEREO Ahead, this is 00:00:00z on March 24, 2015). This implementation simplified removal of the automatic SWAVES power-on in safe mode functionality once normal science operations are restored post-solar conjunction.

\section{Thermal Control}

As part of the load shedding function of each system reset, the FPGA hardware reset sequence disables all spacecraft operational and interface heaters, and enables all spacecraft survival heaters. Because of the long duration of the solar conjunction period, the decision was made to forego this minimum power load configuration, and replicate the thermal control configuration used for long-term science operations so as to moderate any changes to the spacecraft's temperature profile. This configuration consisted of all spacecraft operational and interface heaters enabled, and all spacecraft survival heaters disabled.
The command sequence enabling spacecraft operational and interface heaters and disabling spacecraft survival heaters was added to the absolute timetag/macro command sequences that trigger at solar conjunction entry and exit. Once normal science operations are restored following solar conjunction, these command sequences are removed, as demotions to safe mode are expected to be of relatively short duration.

\section{Fault Protection Modifications}

\section{Fault protection Modifications for Solar Conjunction}

For the solar conjunction mission phase, fault protection established the following phases of attitude control for spacecraft safety and effective communications, see Figure 8. Phase 2 entry and exit is controlled by absolute timetags stored in the on board startup sequence in EEPROM corresponding to the $2^{\circ} \mathrm{SPE}$ entry/exit points.

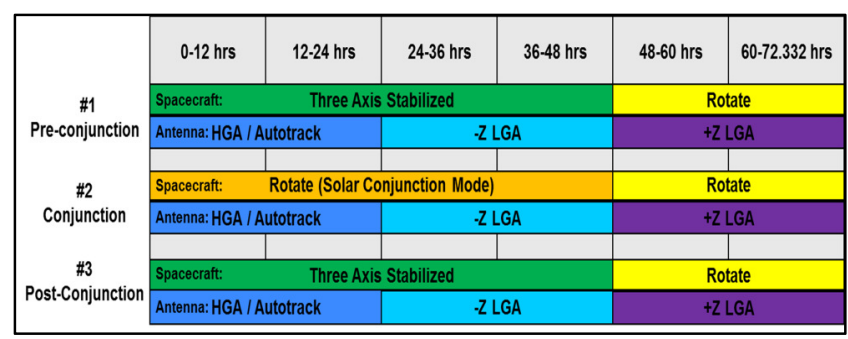

Figure 8. Fault Protection phases of attitude control and RF antenna configuration for solar conjunction mission phase

Fault protection changes necessary for maintaining the health and safety of observatories during the two to three month long solar conjunction periods consists of the following:

- Providing thermal protection for the HGA feed assembly (this protection is via G\&C parameters, not autonomy rules)

- Preserving the limited remaining life of the surviving IMU

- $\quad$ Protecting against harmful effects upon an IMU failure

- Minimizing system momentum accumulation

HGA thermal protection was implemented with off-pointing and using the first two side lobes for communications. The HGA soft stop parameter provides further protection by preventing the feed assembly from ever looking too close to the Sun. It is set by a G\&C parameter in EEPROM as follows:

- $\quad$ Ahead $=8.1^{\circ}$ 
- $\quad$ Behind $=6.8^{\circ}$

The use of the second side lobe for HGA communications during entry and exiting of solar conjunction is controlled by the HGA angle bias G\&C parameter which is set to the following in EEPROM:

- $\quad$ Ahead $=6.1^{\circ}$

- $\quad$ Behind $=6.2^{\circ}$

The flight software computes the HGA rotation angle using the current SPE angle and these two GC parameters as follows:

HGA angle $=$ larger of (SPE angle + HGA angle bias) and HGA soft stop

The limited remaining IMU lifetime is maintained by:

- $\quad$ powering the IMU off immediately after a system reset

- powering-on the IMU if the ST is unhealthy or "lazy"

- powering on the IMU if the flight software determines that an autonomous momentum dump is necessary

- powering off the IMU two hours after the star tracker is locked and operating nominally

To protect against a persistent IMU failure, a G\&C software patch is applied by the on board startup sequence which will prevent bad IMU data from being used by the G\&C application.

\section{GUIDANCE AND CONTROL SUBSYSTEM MODIFICATIONS}

A very small set of $\mathrm{G} \& \mathrm{C}$ parameters were changed to directly support the solar conjunction configuration. Those parameter changes include:

- Earth \& Spacecraft ephemerides - Due to the possibly very long conjunction period (it was unknown in advance at what SPE angle communications would significantly degrade), long-duration spacecraft and Earth orbital information was required to be uploaded to the spacecraft so that the spacecraft can point the HGA at the Earth when it exits conjunction. These ephemerides, which usually cover a three (spacecraft) month or six (Earth) month period, were uploaded to cover out to early February, 2016, which was felt to be the absolute worst-case blackout period. Heading into solar conjunction, these parameters were routinely updated as long as an adequate communications link was available up until the conjunction period.

- HGA pointing parameters - Described above in section 6 .

These were the only G\&C parameter changes explicitly required to support solar conjunction operations.

As a result of the anomalous Behind spacecraft loss of communications during the on-orbit solar conjunction testing on October 1, 2014, a few additional G\&C parameter changes were made the most significant of which was to change the maximum amount of time that an autonomous momentum dump is allowed to complete. At launch this value was set to seven minutes and it had not been changed since all momentum dumps during the mission had been initiated via ground command. Commanded momentum dumps have typically taken 3-4 minutes to complete, and so 7 minutes is certainly quite a bit longer than should be necessary. Further, commanded momentum dumps typically bias the momentum in the opposite direction so that momentum goes through zero and then back up again. This minimizes the science-disrupting dump occurrences. For spacecraft-initiated autonomous dumps, however, the targeted angular momentum is always zero, and so a momentum dump time-out of approximately half (95 seconds) of the normal dump time will enable the majority of momentum to be removed from the system should an autonomous dump be required during solar conjunction. (Note that with the spacecraft rotating during conjunction, momentum accumulation is minimized and few, if any, autonomous dump should be needed.)

\section{Ground System Configuration}

Several ground system configuration changes are necessary for solar conjunction and side lobe operations. We selected the DSN 70 meter track coverage with daily three hour duration to ensure observatory health and safety. The 70 meter stations provide 3 and $10 \mathrm{kbps}$ downlink rates for reduced real-time science during side lobe operations. When the 70 meter stations are not available, the 34 meter stations are used to provide additional coverage at the 633 bps downlink rate, which further limits real-time science and observatory state of health checks. The extra 34 meter tracks increase the science return and allow the operations team to command the observatory daily.

During side lobe testing, insufficient ranging margin was measured on the Ahead and Behind observatories for both side lobes, which indicated ranging data would be intermittent operationally. The Flight Dynamics Facility (FDF) at GSFC determined that it could meet the ephemeris accuracy requirements (three sigma six month prediction) using Doppler data only. As a result, the DSN adjusted $\mathrm{T} 1 / \mathrm{T} 2$ ranging settings in the nominal sequence of events, to 
decrease ranging measurement sampling from one per minute to once every six minutes to decrease ranging dropouts.

The track automation in the MOC performs operator functions for routine ground system operation and observatory commanding, health and safety checks. Track automation modifications were made to conduct operations more efficiently with the reduced track durations and telemetry rates. With these modifications, unattended commanding starts 35 minutes earlier instead of waiting for the usual round trip light time (RTLT) verification of the uplink sweep. To avoid commanding problems caused by solar interference, the MOC changed all commanding to use the COP-1 bypass mode, which prevents communications operation procedure-1 (COP-1) sequence number failures due to dropped commands. The beginning of track (BOT) test command was increased to 10 commands (subsequently to 30 due to the effects of solar interference) to ensure the observatory received commands daily to prevent the command loss timers (CLT) from expiring, which would have complicated operations with contingency recovery for system resets and low gain antenna usage. The automated telemetry checks were modified to account for the reduced telemetry rates and the pre/post conjunction observatory configurations.

The operational procedures and scripts were revised and new ones were created for side lobe and solar conjunction entry/exit operations. The entry and exit procedures keep the operations team organized during critical operations, such as HGA angle bias off-pointing, instrument power down/up, and the observatory system reset into the conjunction phase. The standby mode reset recovery procedure was modified to handle the new system reset startup macro and post-conjunction configuration changes, which is vital for returning the observatory to operational mode and science collection promptly. New templates for the Scheduler planning software were created to plan events for side lobe track operations. Creating side lobe templates instead of making significant changes to existing operational templates helped the team transition back efficiently to planning for routine track operations. All procedures and scripts are tested on the hardware-in-the-loop (HIL) simulator before operational use.

\section{Ground System Testing}

STEREO utilizes two HIL simulators, designated HIL-A (Ahead) and HIL-B (Behind). Each incorporate elements of an engineering model avionics to create a "spacecraft on the ground" that provides a high fidelity testbed for spacecraft simulations. To emulate "missing parts' of the spacecraft, the STEREO Testbed System (STSS) was utilized. The STSS also contains models to simulate the operational environment (orbit, attitude, etc.). Each HIL is controlled from its own workstation via the commercial off-the-shelf
(COTS) command and control application, EPOCH 2000, the same command and control system used in the MOC.

Once created, a new memory object release is first loaded to RAM on the HIL, then dumped, verifying its integrity via cyclic redundancy check (CRC) (unit testing). Functional testing then commences. Depending upon the nature of the change, some functional verification can be more challenging than others. If, for instance, the object release contains a new or updated parameter value, the individual parameter could easily be dumped and visually verified. A new or updated macro would require the user to execute the macro and then check appropriate system logs to verify command timing and execution. An updated autonomy rule would require the user to further configure the system to 'trip' the autonomy rule and verify that the premise and response acted as anticipated (visual and system log verification). Once the object release passes functional testing, it is loaded to EEPROM on the HIL. The HIL setup scripts would also be updated to include the call to the new configuration verification script. At this point the new object release can be scheduled for loading to the actual spacecraft, first to RAM, then to EEPROM two weeks later.

Test plans were created for solar conjunction system level tests. These provided test scenarios, including test set-up instructions and pass/fail criteria. Test activities were summarized in a spreadsheet - test objective, test start/stop times, detailed test steps, test issues, anomalies etc. Archived test data was available to the engineering team shortly after each test for a further, more detailed review. A discussion of significant test results took place at weekly solar conjunction meetings. The following is a list of key tests run on the HIL in preparation for solar conjunction:

- Post-Reset, Initial Condition Verification Test(s)

- $\quad$ Post-Phase 2 System Reset Test

- $\quad$ Soft Command Loss Timer Test(s)

- $\quad$ Expired Ephemeris Test

- Autonomous Momentum Dump Test(s)

- Momentum Dump Without An IMU Test

- $\quad$ Lower Star Tracker TEC Temperature Test(s)

- $\quad$ IMU Code Patch Test(s)

- Operational Longevity Test(s)

On-obit solar conjunction testing includes resetting the actual spacecraft into the solar conjunction configuration (most solar conjunction configuration items are dormant, in EEPROM, only activated through a system reset). The spacecraft then remains in this configuration for 72.332 hours, during which time it cycles through each of the three antenna configurations, and then autonomously resets 
following expiration of the hardware command loss timer. A simulation of this timeline was executed on the HIL prior to the on-orbit test. The HIL was first configured to current on-orbit settings, and then transitioned to the solar conjunction configuration via a system reset, where it remained for the next three days. During this time, anticipated antenna switches were observed along with other key milestones. Following the test, data was accessed from the archive by the engineering team to analyze system performance throughout the three day period. This system level longevity test was instrumental in ensuring that the memory objects and planned sequences would execute as expected on the spacecraft.

\section{ON ORBIT OBSERVATORY TESTING AND LOSS OF COMMUNICATIONS WITH STEREO BEHIND}

The solar conjunction mission phase requires extensive changes to both spacecraft configurations. The potential for errors and unintended consequences is significant. Therefore a robust testing effort is required.

The STEREO operations team maintains a fairly high fidelity hardware-in-the-loop (HIL) simulator for testing spacecraft command sequences on the ground. While much of the spacecraft is simulated in software, the simulator contains replicas of the spacecraft's flight processors and portions of the power subsystem. Thus the simulator runs the actual flight software on identical hardware. Such simulators are sometimes called "flat-sats." This project refers to it as the "HIL."

All configuration changes (parameter values, stored commands, autonomy policies and responses) made for solar conjunction were tested on the HIL. But is this sufficient? The project determined that it was not. For example, the HIL cannot fully simulate an HCLT system reset. Therefore it was decided to conduct controlled tests on each spacecraft.

Six days were requested from the science teams to test the solar conjunction operations as it will occur for each observatory. In summary, using the DSN, the procedures for entry into solar conjunction, 3 days for the HCLT initiated system reset to occur, exiting of solar conjunction, and returning the instrument suites to nominal science data return were tested on orbit. The daily detailed test sequence of events for each observatory is shown in Tables 4 and 5.

Table 4. Ahead On-Orbit Observatory Testing of Solar Conjunction Configurations

Sunday, July $6^{\text {th }}, 1420 z$, Day 1 - Pre conjunction configuration

$800 \mathrm{z}$ - Power off instruments via timetag commands
$>$ Playback SSR

$>$ Switch to HGA $1^{\text {st }}$ side lobe before end of track (EOT)

Monday, July $7^{\text {th }}$, 0530z, Day 2 - Momentum dump on HGA $1^{\text {st }}$ side lobe \& reset into solar conjunction configuration

Acquisition of signal (AOS) using HGA $1^{\text {st }}$ side lobe, $10 \mathrm{kbps} / 500 \mathrm{bps}$

$>$ MOps planned momentum dump with IMU on Set target for conjunction period entry

$>$ System reset (CCD_SYSTEM_RESET) - Test phase 1 configuration (3-axis)

Tuesday, July $8^{\text {th }}, 0550 z$, Day 3 - Monitor solar conjunction configuration - phase 1 ( 3 axis)

AOS using HGA $2^{\text {nd }}$ side lobe, 633 bps/125bps

Wednesday, July $9^{\text {th }}$, 0105z, Day $4-$ Monitor solar conjunction configuration - phase 2 (rotate)

$>$ AOS using HGA $2^{\text {nd }}$ side lobe, 633 bps/125bps

$>$ Reset +41 hours $=$ Test MET timetag to rotate macro 478 (phase 2)

$>$ Reset +48 hours $=$ switch to $-\mathrm{Z}$ LGA (phase 2$)$ (occurred outside of DSN track)

$>$ Reset +60 hours $=$ switch to + Z LGA (phase 2$)$ (occurred outside of DSN track)

Thursday, July $10^{\text {th }}, 0015 z, 0930 z, \& 2155 z$, Day 5 - HCLT firing - phase 2 (rotate) \& Post conjunction configuration

$>\mathrm{AOS}$ using $+\mathrm{Z}$ LGA rotating, $12 \mathrm{bps} / 7.8 \mathrm{bps}$

$>$ Reset +72 hours $=$ HCLT fires, $\mathrm{S} / \mathrm{C}$ resets

$>$ Reset +10 hours $=$ Test UTC timetag fire to stop rotation macro 479 (occurred outside of DSN track)

$>$ AOS using HGA $2^{\text {nd }}$ side lobe, 633 bps/125bps

$>$ Switch HGA to offpoint $1 \mathrm{deg}$ on main lobe

$>$ Playback SSR \& Reconfigure SSR for science operations recording

Friday, July $11^{\text {th }}, 0530 z$, Day 6 - Power on instruments

AOS using HGA offpoint $1 \mathrm{deg}, 360 \mathrm{kbps} / 2000 \mathrm{bps}$

$>$ Reset recovery \& load timetags

Power on all instruments 


\section{Switch to HGA main lobe before EOT}

Saturday and Sunday, Days $7 \& 8$ - Return to nominal science operations

The actual events tested for the solar conjunction mission phase are highlighted in yellow in the timelines in Figures 6 and 7. Note that for comparison, the timeline scale is the same for both observatories. Issues were tracked and anomaly reports and change requests were generated as necessary. A post-test review of results and issues was held.

In July 2014, a week was spent conducting realistic solar conjunction testing on STEREO Ahead. The tests did indeed uncover several minor issues with the configuration changes. Adjustments were made accordingly, and then we conducted a similar test on STEREO Behind in late September 2014.

\section{Table 5. STEREO Behind On-Orbit Observatory Testing of Solar Conjunction Configurations}

Saturday, Sep $27^{\text {th }}$, 1550z, Day 1 - Pre conjunction configuration

$>1800 \mathrm{z}=$ Power off all instruments except SWAVES via timetag commands

$>$ Playback SSR

$>$ Load momentum dump, \#67, for ignition@1630z the next day

Sunday, Sep $28^{\text {th }}, 1530 z$, Day 2 - Momentum dump on HGA $1^{\text {st }}$ side lobe $\&$ reset into solar conjunction

$>$ AOS using HGA $1^{\text {st }}$ side lobe, $10 \mathrm{kbps} / 500 \mathrm{bps}$

$>1630 \mathrm{z}$ - Momentum dump with IMU on - Target set for conjunction period entry, $8 \mathrm{Nms}$

$>1730 \mathrm{z}$ - System reset (CCD_SYSTEM_RESET) Test phase 2 configuration (rotating), SWAVES On

Monday, Sep 29 $9^{\text {th }}$ 0100z, Day 3 - Monitor solar conjunction configuration - phase 2 (rotate) \& 3 (3-axis)

$>$ AOS using HGA $2^{\text {nd }}$ side lobe, 633 bps/125bps

$>0530 \mathrm{z}=$ Reset +12 hours $=$ Test UTC timetag fire to stop rotation (macro 479) (occurred outside of DSN track)

Tuesday, Sep $30^{\text {th }}$, 0900z, 1615z, Day $4-$ Monitor exiting solar conjunction - phase 3 (3-axis)

$>$ AOS using HGA $2^{\text {nd }}$ side lobe, 633 bps/125bps

$>1730 \mathrm{z}=$ Reset +48 hours $=$ switch to $-\mathrm{Z}$ LGA (phase 3), 3-axis monitored using a $34 \mathrm{~m}$ station
Wednesday, Oct $1^{\text {st }}, 1530 z$, Day 5 - HCLT firing - phase 3 (3-axis) \& Post conjunction configuration

$>0530 \mathrm{z}=$ Reset +60 hours $=$ switch to $+\mathrm{Z}$ LGA (phase 3), rotate (occurred outside of DSN track)

$>\mathrm{AOS}$ using $+\mathrm{Z}$ LGA rotating, $12 \mathrm{bps} / 7.8 \mathrm{bps}=+\mathrm{Z}$ LGA calibration for $\sim 2$ revolutions

$>1750 \mathrm{z}=$ Reset +72 hours $=$ HCLT fires, $\mathrm{S} / \mathrm{C}$ resets

$>$ Reacquire on HGA $2^{\text {nd }}$ side lobe, 633 bps/125bps, 3-axis

$>$ Before EOT, Switch HGA to offpoint 1 deg on main lobe

Thursday, Oct $2^{\text {nd }}, 0045 z, 0805 z, 1535 z, \& 2320 z$ Day 6 Power on instruments \& return to science Ops

$>$ AOS using HGA offpoint $1 \mathrm{deg}, 120 \mathrm{kbps} / 2000$ bps

$>$ Playback SSR \& Reconfigure SSR for science operations recording

$>$ Switch to HGA main lobe before EOT

$>$ Reset recovery \& load timetags

$>1635 \mathrm{z}=$ Power on all instruments

Friday, Oct $3^{\text {rd }}$, Day 7 - Return to nominal science operations

The test on STEREO Behind started with a commanded system reset. This was observed from the ground during a station contact. The ST was slightly "lazy" following the reset. Onboard autonomy rules powered up the IMU to provide body rate measurements while waiting for the ST to achieve lock. The ST achieved lock about 12 minutes later, and onboard autonomy powered off the IMU to preserve its life. While not desired, this behavior was not unexpected because the system reset power-cycles the ST.

The plan then called for sending no commands for 3 days to allow the HCLT to reset the system, just as it would (many times) during solar conjunction. Over the course of those 3 days, several station contacts occurred, allowing mission operators to observe telemetry. From this it was confirmed that the spacecraft was performing exactly as planned.

At the end of those 3 days, on October 1, 2014, after the Behind observatory had completed its on orbit testing, the HCLT-initiated reset was observed during a DSN 70 meter station with the radio science receivers recording. Among the many spacecraft actions taken during a reset are powercycling both the ST and the transponder. The drop in telemetry and the reappearance of the spacecraft carrier signal were observed at exactly the expected times. But a few minutes later, the carrier was lost. 
About 10 days later, DSN radio scientists reprocessed the recorded radio signals from that last contact and were able to pull out a few telemetry packets. From these it was learned the ST had once again been lazy and that onboard autonomy had once again powered up the IMU. Also, it was learned that the $\mathrm{X}$-axis gyroscope had spontaneously failed a few seconds after power-up. Now, if the X-gyro had simply "winked out," the spacecraft probably could have recovered. But, unfortunately, the failure was such that the X-gyro continued to provide rate measurements, and they were noisy and likely biased. Once again, if the measurements were only noisy, the spacecraft would likely have survived. Biased measurements, on the other hand, are life-threatening.

It is believed that the biased measurements led the spacecraft software to command the reaction wheels to respond to a perceived roll rate. Since the rate was false, the software would be unable to null it, resulting in the reaction wheel speeds climbing higher and higher. Eventually, the wheels would saturate, and the flight software would attempt to fire thrusters to slow the perceived rotation rate. Simulations have shown that firing thrusters in the presence of a biased rate measurement would likely make the spin worse.

In short, it is believed that the gyro failure led to the spacecraft spinning up and turning away from the Sun. Within a few hours, the battery would be drained, and the spacecraft would start to freeze. A NASA Failure Review Board was convened and concluded that the loss of communications was due to a simultaneous double failure of $\mathrm{G} \& \mathrm{C}$ sensors. Over time, the spacecraft could thaw and slow down and recommended that the project continue to periodically try to reestablish communications with STEREO Behind ${ }^{[1]}$.

\section{Post Conjunction Assessment}

Post solar conjunction assessment objectives are to maintain the health of the spacecraft bus subsystems, evaluate their performance to collect science data, and ensure optimal performance of the limited lifetime resources. To accomplish this, STEREO mission operations uses an automated performance assessment function. This automated system provides the necessary tools to analyze data for anomaly investigations. Missions operations needed to take into consideration that the solar conjunction blackout period is 60 to 105 days and side lobe duration is 400 to 449 days. The automated assessment system is setup for a daily flow, not for analyzing months of data. With the SSR playback at $3 \mathrm{kbps}$, Ahead would require 126 hours, or 16 days (105 day blackout, 8 hour tracks/day), and Behind would require 72 hours, or 9 days (60 day blackout, 8 hour tracks/day).

The Ahead observatory entered the solar conjunction blackout period with 40 days of spacecraft SSR data to downlink. To ensure optimal performance, mission operations began downlinking all 145 days of data that had been recorded during the conjunction. It took six weeks to downlink all of it in multi-day chunks. Using the existing daily assessment processing modified to allow for $\mathrm{N}$ days processing, 4 or more day's data was processed each day. The processing progress was monitored daily to optimize the days processed until all the backlogged data was processed. The same process was used again starting in November 2015 once the HGA had returned to the main lobe, as the entire contents of the SSR, including all the insitu instrument science data, was played back in 3 to 4 days. As 15 months of science data were being processed, missions operations notified the instruments teams weekly as to progress of Level 0 science data availability.

\section{Post Conjunction Results}

Post superior solar conjunction, the Green Bank Radio Telescope detected the STEREO Ahead downlink signal from the HGA at an SPE angle of $0.4^{\circ}$ on May 30, 2015. The Arecibo Observatory also detected the downlink signal at $0.6^{\circ} \mathrm{SPE}$ angle on June $4^{\text {th }}$. Surprisingly, the downlink signal from the $+Z$ LGA was detected at the $1.2^{\circ} \mathrm{SPE}$ angle on June $19^{\text {th }}$ by the Green Bank Radio Telescope.

On June 26, 2015, the first telemetry was received from STEREO Ahead post superior solar conjunction after the $31^{\text {st }}$ HCLT initiated system reset at a $1.5^{\circ}$ SPE angle. The DSN received a strong downlink signal and telemetry using the Goldstone 70 meter station. The observatory was operating nominally continuing to rotate slowly while communicating on the $2^{\text {nd }}$ HGA side lobe as planned. The HGA feed temperature was within the predicted range at $113^{\circ} \mathrm{C}$. System momentum was at the anticipated level of 8.0 Nms. As occurred during solar conjunction entry, after the $31^{\text {st }}$ HCLT reset, the star tracker was again "lazy" to reacquire a solution causing IMU2 to power on for two hours. One additional anomaly occurred, reaction wheel \#3 was not responding to $\mathrm{G} \& \mathrm{C}$ torque commands. While the observatory continued to rotate nominally and was designed to do so with three reaction wheels, as the observatory was healthy, contingency response to this G\&C anomaly was delayed until July $8^{\text {th }}$ to allow for more reliable communications when the SPE angle reached $2^{\circ}$. Daily starting on June $29^{\text {th }}$, a three hour DSN 70 meter station tracked the Ahead observatory as it continued to switch antennas each day and RF performance was found to be highly dependent on solar activity. Due to solar interference, telemetry frame reception varied widely from 13 to 94 percent on the HGA. While carrier lock was received on the LGAs, telemetry lock was never successful on the LGAs due to the low SPE angle.

At $0000 \mathrm{z}$ on July $7^{\text {th }}$, the Ahead observatory returned to nominal 3-axis attitude control as planned and after the $35^{\text {th }}$ and last HCLT initiated system reset on July $8^{\text {th }}$, post 
superior conjunction recovery operations began with the following significant activities:

- $\quad$ Disabled wheel \#3 from G\&C control

- Increased downlink rate to $3 \mathrm{kbps}$ to flow instrument real-time telemetry (SWAVES only)

- Applied leap second

- $\quad$ Spacecraft bus SSR data playback began

- $\quad$ Loaded and verified track timetags

Over the next few days, instruments were powered on and configured for continuing side lobe operations and spacecraft bus recovery operations were completed. The IMPACT SWEA instruments remained off due to low telemetry bandwidth until the HGA returned to the main lobe. On July $20^{\text {th }}$, all instruments were returned to realtime side lobe science, with the three in-situ instrument suites continuing to record space weather data at one packet per minute. The IMPACT Suprathermal Ion Telescope (SIT) instrument required uplinking of several long calibration tables; due to solar interference on the uplink, this activity was postponed until August $26^{\text {th }}$ after the observatory completely cleared the region of solar interference and the uplink rate increased to $500 \mathrm{bps}$ on the $1^{\text {st }}$ HGA side lobe.

The STEREO spacecraft utilizes a diagnostic feature for recording significant events and anomalies on the SSR for quick assessment. These event and anomaly SSR partitions were downlinked first for quick look analysis and revealed that four soft low voltage shutdown (LVS) events had occurred. As the downlink rate from side lobe operations was very limited, the SSR read pointers were repositioned to playback the housekeeping data from the 12 day period which contained the soft LVS occurrences. After the data was analyzed, the cause of the wheel \#3 not responding to torque commands was isolated to the wheel tripping its internal over speed protection limit during a slew caused by the resetting the observatory while the Earth and Sun vectors were closely aligned. Fault protection recovered the observatory to a safe Sun-pointing attitude each time. Wheel \#3 was restored to nominal G\&C control after power cycling it on July $16^{\text {th }}$.

During the post solar conjunction recovery operations on the HGA $2^{\text {nd }}$ side lobe, July $8^{\text {th }}$ through $20^{\text {th }}$, the command success rate varied from $65 \%$ to $100 \%$ depending on solar activity. During this time, the SPE angle increased from 2.0 to $2.5^{\circ}$. While the downlink signal strength was very good during this time, the success rate for telemetry frames received also varied from $76 \%$ to $99 \%$ using the DSN 70 meter stations.

On August $16^{\text {th }}$, the HGA was positioned to return to the $1^{\text {st }}$ HGA side lobe for communications to increase data rates, $10 \mathrm{kbps}$ on the downlink and 500 bps on the uplink, while preventing overheating of the HGA feed assembly. This increased the HGA feed assembly temperature to $121.9^{\circ} \mathrm{C}$, which was within the predicted range, with the HGA angle at $6.8^{\circ}$, with respect to the spacecraft-Sun line.

On November $9^{\text {th }}$, the Ahead observatory resumed operations using the main lobe of the HGA resulting in a 13 $\mathrm{dB}$ signal increase. The HGA soft stop was set to $7.1^{\circ}$ and the HGA rode along a $1^{\circ}$ offset from the center of the main lobe till December $30^{\text {th }}$ to keep the HGA feed assembly cool. This increased the HGA feed assembly temperature to $119^{\circ} \mathrm{C}$, which was within the predicted range. Even at a $1^{\circ}$ offset, data rates increased significantly, $240 \mathrm{kbps}$ on the downlink and 2000 bps on the uplink. The downlink rate gradually returned to the highest rate, $720 \mathrm{kbps}$, when communication on the center of the HGA main lobe returned on December 28, 2015. The National Oceanic and Atmospheric Administration (NOAA) antenna partners gradually were able to close the RF link on the space weather broadcast in between DSN or ESA tracks.

With the increased downlink rate, the 15 months of in-situ instrument space weather data on the SSR were finally able to be played back. During the next five days, the instruments remained in their side lobe science configurations until the SSR could be reconfigured for nominal daily science operations. After the SSR was played back twice and all science data had been received, the SSR was successfully reconfigured for nominal science operations on November $16^{\text {th }}$. As the spacecraft was not designed for side lobe real-time telemetry, to avoid a software design constraint that would cause real-time telemetry queues to jam, the SECCHI real-time telemetry was minimized for 10 hours in between tracks to ensure that the queues were emptied before returning to the higher data rates necessary for nominal science operations on the HGA main lobe.

On November $17^{\text {th }}$, the Ahead spacecraft bus reconfigurations were completed for returning to nominal science operations. After 454 days of side lobe and solar conjunction operations on Ahead, daily SSR playback, commanding, and tracking operations resumed. The IMPACT SWEA instruments were powered on and all instruments were configured for nominal daily science operations over the next few days. The MOC completed processing of the 15 months of in-situ instrument space weather data with the associated data products on November $26^{\text {th }}$. Daily science data return was gradually restored to prime science levels of 5 Gbits by December $28^{\text {th }}$.

Post conjunction, all instruments resumed nominal data collection with no ill effects from being powered down for several months. The HGA feed assembly temperature gradually returned to within tested operational limits, $<90^{\circ}$ $\mathrm{C}$, with no ill effects from being overheated for over two years. Only one lingering issue resulted from the solar conjunction on the Ahead observatory. G\&C fine pointing losses and the magnitude of the attitude roll error increased slightly post solar conjunction. The SECCHI imaging 
instrument team reported that the magnitude of the attitude roll error was periodically affecting the Heliospheric images causing the post processing star-fitting algorithm to fail. To improve roll error performance, the G\&C X-axis control bandwidth was adjusted. The G\&C Rate Merge capability, which enables rate measurements from all sensors to be combined to form a "better" rate estimate versus picking only one sensor as the source of the angular rate measurement, was also enabled, which reduced the noise on the $\mathrm{X}$-axis angular velocity estimate by $\sim 50 \%$. However, the cause for the changed $\mathrm{G} \& \mathrm{C}$ behavior has not yet been determined. Wear on the reaction wheel bearings seems like a possible explanation.

The STEREO Behind observatory entered superior solar conjunction at the $2^{\circ}$ SPE angle on January 22, 2015. Recovery efforts resumed post solar conjunction on May $4^{\text {th }}$ through June $27^{\text {th }}$, as the spacecraft had cleared solar interference for LGA communications. The NASA Failure Review Board recommendations [1] were implemented consisting of battery state of charge recovery and powering on the downlink carrier. The Green Bank Radio Telescope and the Arecibo Observatory also observed the carrier recovery tracks. Due to Behind's retrograde motion causing it to re-enter the region of solar interference, recovery operations were suspended from June $28^{\text {th }}$ through November $29^{\text {th }}$. The Behind observatory recovery operations resumed on November 30, 2015, using the DSN 70 meter stations to command with the Green Bank Radio Telescope and the Allen Telescope Array also observing the carrier recovery tracks depending on availability. While the Arecibo Observatory also assists, the Behind observatory is only in view April through September.

On the $25^{\text {th }}$ attempt to re-establish communications with STEREO Behind, the DSN detected a strong downlink carrier at 2227 UTC on August 21, 2016 after contact was lost for over 22 months. At a spacecraft range of $2 \mathrm{AU}$, the observatory was found to be rotating slowly about its principal axis of inertia for which the uncontrolled attitude allowed some solar array input and continuous uplink and downlink communications on the LGA at emergency data rates. Over the next 22 continuous days, significant obstacles to recovery were overcome with a collaborative effort of the JHU/APL engineering team, NASA GSFC, DSN, FDF, GSFC Space Science Missions Operations (SSMO) scheduling, and Mission Operations teams. This consisted of:

- Reliably commanding a rotating spacecraft with uncontrolled attitude at a distance of $2 \mathrm{AU}$.

- How to power on the spacecraft that was never designed to be off without collapsing the battery voltage.

- Acquiring telemetry at $35 \mathrm{bps}$ from a spacecraft that is rotating with an uncontrolled attitude.
- Warming a frozen propulsion subsystem with a degraded battery and limited solar array input with an uncontrolled attitude.

- Configuring, loading, and verifying EA, C\&DH, and $\mathrm{G} \& \mathrm{C}$ parameters and macros with very limited telemetry.

- Conducting an autonomous momentum dump in the blind and transitioning to $\mathrm{C} \& \mathrm{DH}$ standby mode and successfully receiving telemetry on the HGA indicating star tracker lock and decreasing system momentum.

However, system momentum level remained above the threshold for re-establishing attitude control with the reaction wheels. Due to the uncontrolled attitude, communication degraded and the last detection of the carrier was on September 23, 2016.

From the last telemetry received on September $18^{\text {th }}$, the main bus voltage is low, 2 out of 11 battery cells are currently not functioning, attitude is uncontrolled, rotating at a $\sim 45$ second period about its principal axis of inertia. Propellant is suspected to be frozen and pressure transducer \#2 is not functioning. EA mode is enabled. The battery charge rate is $\mathrm{C} / 10$. Necessary macro sequences have been tested to allow the peak power tracker in $\mathrm{C} \& \mathrm{DH}$ standby mode to protect the battery. These macro sequences will be loaded to EEPROM when the communications supports longer commands.

Monthly recovery efforts will consist of attempting to power on the transmitter for 30 minutes. If no carrier signal is detected, battery recovery operations will commence which consist of repeatedly sweeping a $3 \mathrm{kHz}$ uplink range and sending commands for maintaining battery state of charge.

\section{Conclusion}

The solar conjunction mission phase for STEREO was not initially required by NASA. Nevertheless, after 8 years post launch and multiple mission extensions, it became necessary to safely conduct superior solar conjunction and to continue science return post conjunction. The significance of this work for future deep space missions, for which a long superior solar conjunction is required, is that the mission must be designed to support it. Specifically, the spacecraft must be able to survive a long communication blackout safely. Also, a parabolic HGA cannot be used for communications on the main lobe within several degrees of Sun. The following lessons were learned for incorporation into future NASA missions.

1. Add value based requirements for the extended mission phase for NASA missions. NASA missions are designed for the duration of the prime science success criteria. They do not reflect the reality for average 
NASA observatory lifetime, which is typically greater than 9 years. During development, value based requirements should be discussed for continuing the mission beyond the prime science phase to include all subsequent mission significant orbital events. For example for STEREO, adding a single requirement for safe survival of the months long superior solar conjunction would have uncovered the following:

- HGA feed thermal issue

- Unmodifiable 3 day HCLT initiated system resets

- Star tracker power off for each system reset

- No RF margin on LGAs at a 2 AU range

2. Don't make the hardware command-loss timer (HCLT) unmodifiable. Rather design the HCLT duration as an uploadable parameter. If this feature had been available, we would have set the timeout value to a large number to prevent the 35 system resets during the blackout period. This action would have prevented the 4 LVS events and the reaction wheel over-speed latchup.

3. Allow the system reset hardware response to be modified post-launch, i.e., EEPROM, not FGPA. For example, STEREO's unmodifiable reset sequence turns the star tracker off. The powering on and subsequent slow star re-acquisition of the star tracker was a contributing cause of the Behind loss of communication anomaly.

4. Set the battery charge current to maximum at start-up power initialization and after going into low state of charge operation to allow maximum charging capabilities with available solar array power. This design change would improve the odds of recovering a spacecraft from a depleted battery condition.

5. If a spacecraft has redundant IMUs, allow for cross strapping between individual units so as to permit full three axis coverage using any combination of sensors capable of providing measurements along three mutually perpendicular axes. Further, individual IMU units should be oriented on the spacecraft in a way that permits maximum flexibility in combining outputs while covering three mutually perpendicular axes.

\section{ACKNOWLEDGEMENTS}

Many thanks to the engineering, mission operations, and management staff for all their dedicated support to analyze, develop, test, and configure the aging STEREO observatories for a mission phase that, while not required by NASA, was necessary to keep the twin Heliophysics data pipelines flowing beyond the lengthy superior solar conjunction.

\section{REFERENCES}

[1] Harman, R.R. et al, "Solar Terrestrial Relations Observatory BEHIND (STEREO-B) Loss of Communications Failure Review Board Report", NASA GSFC, March 20, 2015.

\section{BIOGRAPHY}

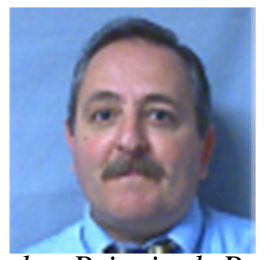

Daniel A. Ossing received a B.S. in Marine Engineering, from the Massachusetts Maritime Academy in 1982 and an M.S. in Electrical Engineering from The Johns Hopkins University in 1989. As a member of the Principal Professional Staff he has been with the JHU/Applied Physics Laboratory for more than 32 years. He has been the mission operations manager of NASA's STEREO mission since 2008. Concurrently, he was also the mission operations manager for the first JHU/APL dual cubesat mission, MBD. He has conducted mission operations for the ISRO Chandrayaan-1, NASA LCROSS, NASA TIMED, and MDA MSX missions. His career started as an Engineering Officer for ARCO Marine transporting Alaskan north slope crude oil.

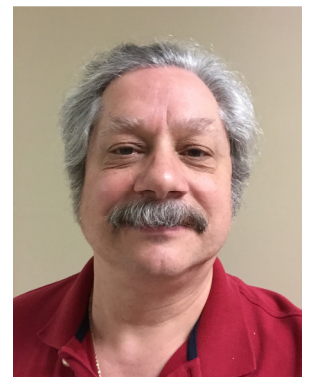

David A. Quinn received a B.S. in Aerospace Engineering from the University of Maryland at College park in 1983 and a M.S. in Mechanical Engineering from George Washington University in 1998. He has been with NASA/GSFC for more than 28 years. His career at GSFC started with leading the Pointing Control System group for the Hubble Space Telescope during the launch, deployment, operation and first servicing mission. He was involved in operations, mission design, analysis, and flight dynamics for the Landsat-4, 5, \& 7, TERRA, LRO, LCROSS, LADEE, and TESS missions. During his time at GSFC, he co-invented the formation flying technique fundamental to multi-spacecraft control efforts at GSFC. He also conceived and developed the CEGANS (Compound Eye GPS Attitude and Navigation Sensor) concept for which he received a U.S. patent in 2003. He authored a proposal for the Cross-Enterprise Technology Development Program (CETDP) for tethered formation flying development and was awarded funding to serve as the Principle Investigator for tethered formation flying development in support of the SPECS mission concept. Currently, he is the Mission Director for the NASA/GSFC Space Science Mission Operations branch where he has primary operations responsibility for the NASA TIMED, STEREO, Van Allen Probes, and SOHO missions. 